\title{
DOENÇA DE WILSON DIAGNÓSTICO CLÍNICO E SINAIS DAS "FACES DO PANDA" À RESSONÂNCIA MAGNÉTICA
}

Ao Editor - O interessante e ilustrativo relato de caso de Brito et al. ${ }^{1}$ é uma contribuição relevante para a divulgação das alterações de neuroimagem observadas na doença de Wilson (DW). Entretanto, algumas considerações sobre este artigo parecem-nos pertinentes.

As citações históricas mencionando as descrições iniciais de Westphal, Strümpell e Wilson enriquecem a descrição do caso. Contudo, é necessário esclarecer que Adolf Strümpell descreveu, em duas publicações ${ }^{2,3}$, três casos de pseudoesclerose, sendo que na primeira, de 1898, em que são relatados os dois primeiros, não há referência à presença de cirrose hepática. É somente na descrição do seu terce i rocaso de pseudoesclerose, numa segunda publicação sobre o tema, no ano de 1899, que Strümpell assim registra a presença de comprometimento hepático: "Von den Befunden an den übrigen inneren Organen sind bemerkenswerth eine beginnende Lebercirrhose und mehrere deutliche alte Narben and den wharen Stimmbändern (offenbar syphilitischer Natur)". En tretanto, como pode ser notado neste trecho do relato do caso, o autor considerou que a presença de cirrose hepática era casual e possivelmente relacionada à sífilis.

Na página 178 os autores referem-se à importância da ceruloplasmina como um dos elementos laboratoriais de fundamental importância e salientam que taxa normal de ceruloplasmina foi registrada em um paciente de Finelli ${ }^{4}$. A normalidade da concentração de ceruloplasmina em pacientes com DW é um dado realmente digno de menção mas, conhecido de longa data e apoiado em vários registros da literatura e não apenas na descrição recente e isolada de Finelli. Assim, já em 1978, Sass-Kortsak e Bearn ${ }^{5}$ observavam que em 230 casos de DW compilados da literatura $4 \%$ apresentavam níveis normais de ceruloplasmina. Mais recentemente, Hoogenraad ${ }^{6}$ refere a ocorrência desse perfil bioquímico atípico para a DW em cerca de $5 \%$ dos casos e Brewer ${ }^{7}$ em $20 \%$.

Na página 179, abordando aspectos terapêuticos do caso descrito os autores mencionam que drogas menos tóxicas como a trietilenotetramina (trientina) e o tetratiomolibdato não estão disponíveis no mercado nacional. A trientina, de fato é uma droga menos tóxica que a penicilamina, contudo, o tetratiomolibdato embora ofereça menor risco de piora neurológica pode ter atividade supressiva sobre a medula óssea manifestada por anemia ou leucopenia, elevação de transaminases e ação tóxica sobreóssos em crescimento, devendo seu uso ser evitado em crianças e adolescentes 7,8 . Devido a esses efeitos colaterais a duração do tratamento com tetratiomolibdato tem sido restringida para 8 semanas e o seu uso para a DW ainda não foi aprovado nos Estados Unidos da América pela Food and Drug Adminitration (FDA).
Outro aspecto a ser destacado é que em pacientes com manifestações neurológicas da DW as imagens de ressonância magnética (RM) mostram alterações de sinal no putâmen, particularmente em T2, com freqüência maior que as alterações observadas no tronco cerebral que caracterizam a "face do panda" 9 . Essas alterações de sinal no putâmen (hipersinal periférico), também p resentes no caso descrito por Brito et al. ${ }^{1}$, além de mais freqüentes que a "face do panda" são igualmente muito sugestivas da DW e as que persistem por tempo mais longo após o tratamento ${ }^{10}$.

\section{REFERÊNCIAS}

1. Brito JCF, Coutinho MAP, Almeida HJF, Nóbrega PV. Doença de Wilson. diagnóstico clínico e sinais das "faces do panda" à ressonância magnética. Arq Neuropsiquiatr 2005;63: 176-179.

2. Strümpell A. Über the Westphal'sche Pseudosklerose und über diffuse Hirnsklerse, insbesondere bei Kindern. Dtsh Z Nervenh 1898;12: 115-149.

3. Strümpell A. Ein weiterer Beitrag zur Kenntniss der sog. Pseudosklerose. Dtsh Z Nervenh 1899;14:348-355.

4. Finelli P. Kayser-Fleischer ring: hepatolenticular degeneration(Wilson's disease). Neurology 1995;45:1261-1262.

5. Sass-Kortsak A, Bearn AG. Hereditary disorders of copper metabolism. In Stanbury JB, Wyngaarden JB, Fredrickson DS (eds). The metabolic basis of inherited disease. New York: Mcgraw-Hill, 1978: 1095-1126.

6. Hoogenraad T. Wilson's disease. Amsterdam: Intermed Med Pub, 2001: 113.

7. Brewer JG. Neurologically presenting Wilson's disease: epidemiology, pathophysiology and treatment. CNS Drugs 2005;19:185-192.

8. Lewitt P, Pfeiffer R. Neurologic aspects of Wilson's disease: clinical manifestations and treatment considerations. In Jancovicks JJ, Tolosa E (eds). Parkinson's disease and movement disorders. Philadelphia: Lippincott Williams \& Wilkins, 2002.

9. Magalhães ACA, Caramelli P, Menezes JR, Lo LS, Bacheschi LA, Barbosa ER, Rosemberg S. Wilson's disease: MRI with clinical correlation. Neuroradiology 1994;36: 97-100.

10. Costa MDL, Barbosa ER, Leite CC, Bahia VS, Camargo ECS, Bacheschi LA. Neuroimaging follow-up of patients with Wilson's disease under treatment: report of fourteen cases. Neurology 1999 (Suppl 2); 52: A224-225.

Egberto Reis Barbosa, Livre-Docente Alexandre Aluízio Costa Machado, Pós-Graduando Departamento de Neurologia da Faculdade de Medicina da Universidade de São Paulo

Resposta dos Autores - Agradecemos ao doutor Egberto Reis Barbosa e ao pós-graduando Alexandre Aluizio Costa Machado o interesse pelo nosso artigo ${ }^{1}$. Pelas críticas construtivas recebidas evidenciamos que alguns dados não foram aceitos ou deram margens a interpretações que não traduzem o sentido realmente pretendido, necessitando, portanto, os devidos esclarecimentos.

O espaço limitado oferecido pelo periódico obri- 
gou-nos a discorrer com mais detalhes o que consideramos objetivos do trabalho: diagnóstico clínico e os sinais das "faces do panda" mostrados pela ressonância magnética (RM). Assim sendo, pode-se compreender a maneira bastante resumida como foram descritos os fatos históricos e as medidas terapêuticas.

Em relação ao trabalho de Strümpell, não nos opomos à correção. Cumpre ressaltar que a citação do ano de 1898 baseou-se em McDowell et al. ${ }^{2}$ cujo texto referia: "In 1898 Strümpell reported 2 patients with tremor in whom liver disease was found at postmortem examination".

No que diz respeito à concentração normal de ceruloplasmina na doença de Wilson (DW) referida por Finelli, nossa intenção, de cunho puramente ilustrativo, foi mostrar mais um dado que pode dificultar o diagnóstico da doença, mas sem qualquer alusão histórica como, supomos, os senhores deduziram.

O paciente estudado por nós é portador de sintomatologia neurológica acentuada. O tratamento inicial com acetato de zinco não apresentou resposta satisfatória. A introdução da penicilamina, em substituição, provocou efeitos colaterais que motivaram sua suspensão após 40 dias de uso. Daí, a referência ao tetratiomolibdato, droga inexistente no mercado nacional, de ótima ação quelante e que não provoca piora do quadro neu rológico. As complicações hematológicas realmente existem mas podem ser revertidas com a suspensão do medicamento ${ }^{3}$. Ademais, supressão de medula óssea também faz parte dos efeitos adversos da penicilamina. Por outro lado, drogas não liberadas pela Food and Drug Administration (FDA) existem no mercado nacional e são de uso corrente como, por exemplo, a dipirona e a flunarizina. Certamente, estes esclarecimentos não significam que defendemos o uso do tetratiomolibdato no tratamento da DW, pois trata-se de uma droga que conhecemos apenas através da literatura.
Quanto aos achados da RM na DW, levantamos as seguintes considerações:

1) As imagens de hiperintensidade periférica em $T 2$ observadas no putâmen são, de fato, importantes mas não apresentam especificidade e podem ser encontradas na região central do núcleo ${ }^{4}$ ou difusamente ${ }^{5}$;

2) O sinal da "face do panda" é considerado característico da DW4 e sua baixa freqüência não está devidamente estudada, haja vista tratar-se de imagem radiológica divulgada pela primeira vez em $1991^{4}$ e somente 9 anos depois re gis trourse outro relato ${ }^{6}$. Esta lacuna temporal pode depender, portanto, de vários fatores como baixa freqüência, desconhecimento da existência do sinal ou mesmo pelo desaparecimento da imagem durante o tratamento. Ademais, o nosso trabalho registra o primeiro caso da literatura nacional, fato que consideramos relevante e que motivou o envio para publicação;

3) Finalmente, consideramos ainda que o diagnóstico da DW baseia-se, sobretudo, na sintomatologia, nas alterações da ceruloplasmina e do cobre e na evidência do anel de Kayser-Fleischer.

\section{REFERÊNCIAS}

1. Brito JCF, Coutinho MAP, Almeida HJF, Nóbrega PV. Doença de Wilson: diagnóstico clínico e sinais das "faces do panda" à ressonância magnética. Relato de caso. Arq Neuropsiquiatr 2005;63:176-179.

2. McDowell FH, Lee JE, Sweet RD. Extrapiramidal disease. In Baker AB (ed). Clinical neurology. Philadelphia: Harper \& Row, 1978; 38: 1-67

3. Brewer GS. Wilson's disease. Curr Treat Opt Neurol 2000;2:193-203.

4. Hitoshi S, Makoto I, Yoshikava K. Mid-brain pathology of Wilson's disease: MRI analysis of three cases. J Neurol Neurosurg Psychiatry 1991; 54:624-626.

5. Neto M Jr. Doenças da substância branca e dos núcleos da base. In Magalhães ACA. Ressonância magnética do sistema nervoso central. São Paulo: Editora Atheneu, 1999: 125-150.

6. Kuruvilla A, Joseph S. "Face of giant panda" sign in Wilson's disease: revisited. Neurol India 2000;48:395-396.

José Correia de Farias Brito Paulo Virgolino da Nóbrega

\section{DEMÊNCIA SEMÂNTICA}

Ao Editor - Como é minha rotina, li com atenção os artigos dos Arquivos de Junho de 2005, entre eles “Demência semântica: avaliação clínica e de imagem. Relato de caso. Arq Neuropsiquiatr 2005;63:348-352", de L. Caixeta e L.L. Mansur.

Eu gostaria de cumprimentar os autores pela qualidade da apresentação do caso, e, respeitosamente, sugerir que sua afirmativa de que não existem "casos autóctones" seja corrigida. Nós publicamos um caso semelhante há 16 anos, nos primórdios internacionais dos conceitos sobredemências frontais, quando ainda se discutiam os limites exatos de doença de Alzheimer (Oliveira SAV, Cast roMJMO, Bittencourt PRM. Slowly prog ressive aphasia followed by Alzheimer's dementia: a case report. Arq Neuropsiquiatr 1989;47:72-75). O caso era autóctone: o paciente pertencia a uma tradicional família brasileira. Da mesma maneira, a afirmação sobre o "relativo desco- nhecimento desta forma de demência no Brasil", precisa ser revista.

Imagino que nosso artigo não tenha sido revisado pelos autores pela sua antigüidade. Pode até ser um elogio indireto, que nossa publicação tenha sido muito precoce para um artigo nacional: seria o quarto mais antigo da revisão bibliográfica dos autores, publicado 7 anos após a descrição original de Marcel Mesulam. Segundo discussão em recente congresso brasileiro de neurologia, após uma mesa redonda sobre demências f rontotemporais (Nitrini R, comunicação pessoal, 2002), nossa publicação fez a primeira menção à doença de Alzheimer na literatura neurológica brasileira. Portanto, faz parte da história dos estudos das demências no Brasil. É claro, passou mesmo crivo editorial que o artigo de Caixeta e Mansur.

$\mathrm{O}$ artigo original contém uma tomografia computa- 